\title{
$\boldsymbol{N} \times \boldsymbol{N}$ 非等谱 AKNS 族的李代数结构
}

设 $V_{0}=\operatorname{diag}\left(d_{1}, \cdots, d_{N}\right), V_{1}=((1-$ $\left.\left.\delta_{i j}\right) u_{i j}\right)$, 其中 $d_{i}$ 为互异复常数, 位势 $u_{i j}=$ $u_{i j}(x, t)$ 对固定的 $t$ 取自 Schwartz 空间 $S$. 记 $x^{0}=1, x^{1}=x$ 并确定矩阵 $W_{k}^{a}$, $(k \geqslant 0, a=0,1)$ 如下: $W_{0}^{a}=x^{a} V_{0}, W_{1}^{a}=$ $x^{a} V_{1}, a \delta_{j 0} V_{0}-W_{i x}^{a}+\left[\begin{array}{ll}V_{0}, & W_{i+1}^{a}\end{array}\right]+\left[V_{1}\right.$, $\left.W_{i}^{a}\right]=0, i \geqslant 0$ 及 $\rho\left(W_{k}^{a}\right)=k-a$, 这里权 $\rho$ 定义为 $\rho\left(u_{i j}\right)=\rho(\partial / \partial x)=-\rho(x)=1$. 我们称方程族 $V_{1 s_{n}^{a}}=\left[V_{0}, W_{n+1}^{a}\right]$ 为 $N \times N$ 非等谱 AKNS 族,对之我们证得

定理 $1 d W_{k}^{a} / d t_{n}^{b}=F_{b, n}^{a, k}$, 其中

$$
F_{b, k}^{a, k}=a(k-1) W_{n+k-1}^{o}
$$

$$
+\sum_{s=0}^{k-1}\left[W_{s}^{a}, W_{n+k-s}^{b}\right\}
$$

\section{定理 2}

$\sum_{s=0}^{k}\left[W^{a}, W_{k-s}^{0}\right]=(b-a)(k-1) W_{k-1}^{k}$.

\section{定理 3}

$$
\begin{gathered}
\left(d / d t_{n}^{b}\right) F_{a, m}^{c, k}-\left(d / d t_{m}^{a}\right) F_{b, n}^{c, k} \\
=(m b-n a) F_{w, m+n-1}^{c, k} .
\end{gathered}
$$

当 $c=0, k=1$ 及 $N=2$ 时定理 3 给 出了李翊神和朱国城发现的无穷维李代数结 构 (J. Phys A, 即将发表)。

屠规彰 (中国科学院计算中心，北京）

\section{树的几个计数问 题}

对于 $t \geqslant 2$ 叉树和有序树, 具有 $r \geqslant 0$ 棵非空子树的节点称为 $r$ 次节点. 考虑具有 $n \geqslant 0$ 个节点的 $\iota$ 叉树族 $\Gamma_{n}$ 和有序树族 $Q_{n}$, 本文建立了以下结果. 它们在计算机科学的 算法分析中有着直接的应用.

定理 1 设 $b_{n}$ 是 $\Gamma_{n}$ 中树的总数, $g_{k, n}^{1, n}$ 是 $\Gamma$ ，中位于第 $k$ 层的 $r$ 次节点的总数,并且

$$
B(y)=\sum_{n=0} b_{n} y^{*}, G^{[n]}(z, y)=\sum_{k, n>0} z^{k} y^{*},
$$

则

$$
\begin{gathered}
G^{(n)}(z, y)-\frac{1}{1-t y z[B(y)]^{z-1}} \\
\cdot\left(\begin{array}{c}
t \\
r
\end{array}\right) y(B(y)-1]^{r} \\
g_{k, n}^{(r)}=\frac{t r+(t-1) k}{t(n-1)-k}\left(\begin{array}{l}
t \\
r
\end{array}\right)
\end{gathered}
$$

$$
\cdot\left(\begin{array}{c}
t(n-1)-k \\
n-r-k-1
\end{array}\right) t^{k}, \quad g_{0.1}^{(0)}=1 .
$$

定理 2 设 $a_{n}$ 是 $O_{n}$ 中树的总数， $d_{k}^{[r]}$ 是 $O_{n}$ 中位于第 $k$ 层的 $r$ 次节点的总数,并且

$$
\begin{aligned}
A(y) & =\sum_{n=0} a_{n} y^{n}, \\
D^{[r]}(z, y) & =\sum_{k, n>0} d_{k, n}^{|r|} z^{k} y^{n},
\end{aligned}
$$

则

$$
\begin{gathered}
D^{|r|}(z, y)=\frac{y^{2}[A(y)]^{r}}{y-z[A(y)]^{2}}, \\
d_{k, n=}^{r+1}=\frac{2 k+r}{2(n-1)-r}\left(\begin{array}{c}
2(n-1)-r \\
n+k-1
\end{array}\right), \\
d_{0.1}^{[0.1}=1 .
\end{gathered}
$$

由上述定理可导出许多特殊结果，特别 地, 我们有

推论 1 设 $g_{n}^{[r]}$ 是 $\Gamma_{n}$ 中 $r$ 次节点的总 
数， $g_{k, n}$ 是 $\Gamma_{n}$ 中位于第 $k$ 层的节点总数， $f_{n}^{[r]}$ 是 $\Gamma_{n}$ 中 $r$ 次节点的总通路长度, 并且 $n>1$, 则

$$
\begin{gathered}
g_{n}^{[r]}=\left(\begin{array}{c}
t \\
r
\end{array}\right)\left(\begin{array}{c}
t(n-1) \\
n-r-1
\end{array}\right), \\
g_{k, n}=\frac{t+(t-1) k}{t n-k}\left(\begin{array}{c}
t n-k \\
n-k-1
\end{array}\right) t^{k}, \\
f_{n}^{[r]}=\left(\begin{array}{l}
t \\
r
\end{array}\right) \sum_{i=r+1}^{n-1}\left(\begin{array}{c}
t(i-1) \\
i-r-1
\end{array}\right)\left(\begin{array}{c}
t(n-i) \\
n-i
\end{array}\right) .
\end{gathered}
$$

推论 2 设 $d_{n}^{(r)}$ 是 $Q_{n}$ 中 $r$ 次节点的总 数, $d_{k, n}$ 是 $\Omega_{n}$ 中位于第 $k$ 层的节点总数, $e_{n}^{[r]}$
是 $Q_{n}$ 中 $r$ 次节点的总通路长度, 并且 $n>$ 1 , 则

$$
\begin{gathered}
d_{n}^{(r)}=\left(\begin{array}{c}
2 n-r-3 \\
n-2
\end{array}\right), \\
d_{k, n}=\frac{2 k+1}{2 n-1}\left(\begin{array}{c}
2 n-1 \\
n-k-1
\end{array}\right), \\
e_{n}^{(r)}=\sum_{i=r}^{n-2}\left(\begin{array}{c}
2 i-r \\
i
\end{array}\right)\left(\begin{array}{c}
2(n-2-i) \\
n-2-i
\end{array}\right) .
\end{gathered}
$$

王振宇 孙湖义 （武汉数字工程研究所）

\section{关于 Leavitt 和 Rossa 的两个问题}

对任一环 $R$, 令 $F(R)=\{A \mid$ 若 $0 \neq$ $I \triangleleft \triangleleft A$, 则有 $J \triangleleft \triangleleft I$ 使 $J \sim R\}$, 这里 $I \triangleleft \triangleleft A$ 表示 $I$ 是 $A$ 的次理想. 并记 $R^{0}$ 为环 $R$ 上的零环. 易知 $\mathbf{Z}^{0} \in F\left(\mathbf{Z}_{n}^{0}\right), \mathbf{Z}_{p}^{0} \in F\left(\mathbf{Z}_{p}^{0}\right)$, 其中 $\boldsymbol{p}$ 为素数, $n$ 为任意自然数,

$$
\mathbf{Z}_{n}=\mathbf{Z} / n \mathbf{Z} \text {. }
$$

最近 Leavitt 和 Rossa ${ }^{[1]}$ 在研究根类的极 小生成元的问题中提出了下面两个问题:

问题 1. 对于一个域 $K, F\left(\sum_{1}^{\infty} K^{0}\right)$ 是否等于零? 这里 $\sum_{1}^{\infty}$ 表可数无限直和.

问题 2. 对于合数 $n$, 除了 $\mathbf{Z}^{0}, F\left(Z_{n}^{0}\right)$ 是否含别的(非零)环?

本文给出两个定理. 定理 1 否定回答了 问题 1 , 定理 2 肯定回答了问题 2 .

定理 1 设 $K$ 是一个域,则

$$
F\left(\sum_{1}^{\infty} K^{0}\right) \neq 0 \text {. }
$$

证 取符号集 $E=\left\{e_{\alpha} \mid \alpha \in Q, \alpha>\right.$ 0\}, 规定

$$
e_{\alpha} e_{\beta}=e_{\alpha+\beta},
$$

则 $E$ 是一个可换半群. 作 $K$ 代数

$$
A=K[E]=\left\{u_{1} e_{\alpha_{1}}+u_{2} e_{\alpha_{2}}\right.
$$

$$
\begin{gathered}
+\cdots+u_{n} e_{a_{n}} \mid \\
0 \neq u_{i} \in K, 0<\alpha_{i} \in \mathbf{Q},
\end{gathered}
$$

且可设

$$
\left.a_{1}<\alpha_{2}<\cdots<\alpha_{n}\right\} .
$$

按照分配律定义 $A$ 中任二元素的乘积, 则 $A$ 成为一个(可换)环. $\forall 0 \neq a \in A$, 设

$$
\begin{aligned}
& a=u_{1} e_{a_{1}}+u_{2} e_{\alpha_{2}}+\cdots+u_{n} e_{a_{n}}, \\
& 0 \neq u_{i} \in K, \quad \alpha_{1}<\alpha_{2}<\cdots<\alpha_{n},
\end{aligned}
$$

则称 $u_{1} e_{a_{1}}$ 是 $a$ 的首项. 易知 $a^{2}$ 的首项是 $u_{1}^{2} e_{2 \alpha_{1}}(\neq 0)$, 所以 $a^{2} \neq 0, A$ 是半素环. 下 证

$$
A \in F\left(\sum_{1}^{\infty} K^{0}\right) .
$$

由 Andrunakievich 引理, $A$ 的每个非零次理 想都包含一个非零理想,因此要证

$$
A \in F\left(\sum_{1}^{\infty} K^{0}\right),
$$

只需证明 $\forall 0 \neq H \triangleleft A, H$ 有一个次理想

$$
l \sim \sum_{1}^{\infty} K^{\mathrm{i}} .
$$

若 $u e_{\alpha}(u \in K)$ 是 $H$ 中某个元素 $w$ 的首项, 那 么容易看到, 对任意有理数 $\beta>\alpha$ 和 $K$ 中任 意非零元 $\nu, H$ 中(至少)有一个元素,它的 